\title{
Determinants of anxiety and depression in patients with cubital tunnel syndrome
}

\author{
Siming Jia ${ }^{1,2+}$, Xiaoying Shi ${ }^{2,3 \dagger}$, Guanglian Liư ${ }^{4}$ Li Wang ${ }^{1}$, Xiaoran Zhang ${ }^{1}$, Xuelin Ma', Jia Li ${ }^{5}$ and Xinzhong Shao ${ }^{1 *}$
}

\begin{abstract}
Background: The aim of this cross-sectional study to assess the proportions of anxiety and depression in patients with CUTS, and to explore the associated demographic and clinical features.

Methods: From May 2011 to January 2017, 246 patients diagnosed with CuTS were recruited. The Hospital Anxiety and Depression Scale was used to assess the proportions of depression and anxiety. Patient demographic and clinical data were collected. Univariate analysis and multivariate regression were carried out to identify the variables that were independently associated with anxiety and depression.

Results: The proportions of depression and anxiety were 17.9\% $(n=44)$ and 14.2\% $(n=35)$, respectively. Five patients had both possible/probable anxiety and depression. Logistic regression analysis revealed that diabetes mellitus was independently associated with depression; and the modified McGowan grade was independently associated with anxiety.

Conclusions: In patients with CUTS, the proportions of depression and anxiety were 17.9\% and 14.2\%, respectively. Early screening for anxiety and depression is beneficial for patients with CuTS.
\end{abstract}

Keywords: Cubital tunnel syndrome, Anxiety, Depression, Neurophysiology, Hospital based study

\section{Background}

Cubital tunnel syndrome (CuTS) is the second most common peripheral nerve entrapment syndrome, caused by compression of the ulnar nerve within the cubital tunnel at the elbow [1-3]. Patients with CuTS are often troubled by symptoms like tingling and pain in the small finger and ulnar half of the ring finger [4]. As the condition progresses, muscular weakening and muscular atrophy will occur [5]. Owing to these symptoms, patients' quality of life and the ability to continue employment may be impacted.

CuTS and depression are highly prevalent conditions, specifically among women [6]. In the general population, the global prevalence for major depressive disorder is $5 \%$, whereas for anxiety disorders is $4 \%$ [7]. Mood disorders such as depression and anxiety increase the pain perceived and

\footnotetext{
*Correspondence: shaoxzhong@126.com

${ }^{+}$Siming Jia and Xiaoying Shi are co-first authors

'Department of Hand Surgery, The Third hospital of Hebei Medical

University, Shijiazhuang 050000, Hebei, China

Full list of author information is available at the end of the article
}

worsen the functional status [8]. Many published articles focus on disease progression, but few studies on patients' mental status [9]. In a cross-sectional study, Van et al. [10] found patients with disorders on the upper extremity were more frequently reported anxiety and depression. They reported the proportions of depressive and anxiety disorders were 16 and 9\% in patients with CuTS. Therefore, it is important to assess the mental status of patients with CuTS.

The aim of the study was to assess the proportions of anxiety and depression in patients with CuTS and to explore the associated demographic and clinical features.

\section{Methods \\ Participants}

From May 2011 to January 2017, patients diagnosed with CuTS were recruited from the outpatient service of the hospital. The number of variable was used to calculate the optimal number of participants for this study. In this study, the sample size was typically expressed in terms of events per variable. An events per variable of 10 was widely used as 
the low limit for developing the models that predicted a binary outcome [11]. The sampling method was convenient sampling. The diagnosis of CuTS was made using a combination of clinical evaluation and nerve conduction study. Clinical assessments included the history of initial presence with intermittent paresthesias, numbness, and tingling in the small finger and ulnar half of the ring finger [4]. According to the guideline of the American Association of Electrodiagnostic Medicine [12], all patients underwent the nerve conduction study. Confirmatory criteria included: (1) motor nerve conduction velocity (MNCV) across the elbow less than $50 \mathrm{~m} / \mathrm{s}$; (2) an MNCV difference of greater than $10 \mathrm{~m} / \mathrm{s}$ between the elbow segment and forearm segment; (3) a conduction block with compound muscle action potential decreased more than 20\% (amplitude measured from the elbow to upper arm). Electrodiagnostic studies were performed by a specialist technician using a Dantec Keypoint Portable Nerve Conduction/ electromyography machine (Dantec Dynamics, Bristol, Bristol, UK) and reported by a consultant neurophysiologist. The specific process is shown in Fig. 1.

Patients were selected based on one of the following criteria: (1) patients with subjective symptoms, no matter the presence of intrinsic muscle atrophy or not; (2) electrodiagnostic evidence of CuTS; and (3) age > 18 years because the study just focused on adult patients. Patients with one of the following criteria were excluded: (1) age $\leq 18$ years; (2) patients with other neuropathy confirmed electrophysiologically; (3) patients refused to attend the study; (4) patients who had undergone previous treatments, such as splinting, steroid injection, or cubital tunnel release; (4) a previous diagnosis of anxiety, depression, and other psychiatric disorder; (5) pregnant and lactating women because they were at high risks of depression and anxiety that distorted the assessments [13].

\section{Demographics and clinical evaluation}

We used the self-administered questionnaire to assess the patients, which consisted of two parts. The first part included patients' demographic data (age, gender, educational level, marital status, job status, and socioeconomic status). The second part was patients' clinical data (hypertension, diabetes mellitus, tobacco use, alcohol use, history of cancer, and duration of symptoms). Patients were determined as older ( $>50$ years) and younger $(\leq 50$ years) adults. Educational level was registered as university degree, primary and middle degree, and illiterate degree. Marital status was coded as married, single (with or without cohabiting), widow, and divorced. Job status was registered as employed (in vacation or not) and unemployed (including students). Based on the classifying occupation, employed patients were classed into three groups: unskilled group, semi-skilled / skilled group and semi- professional/

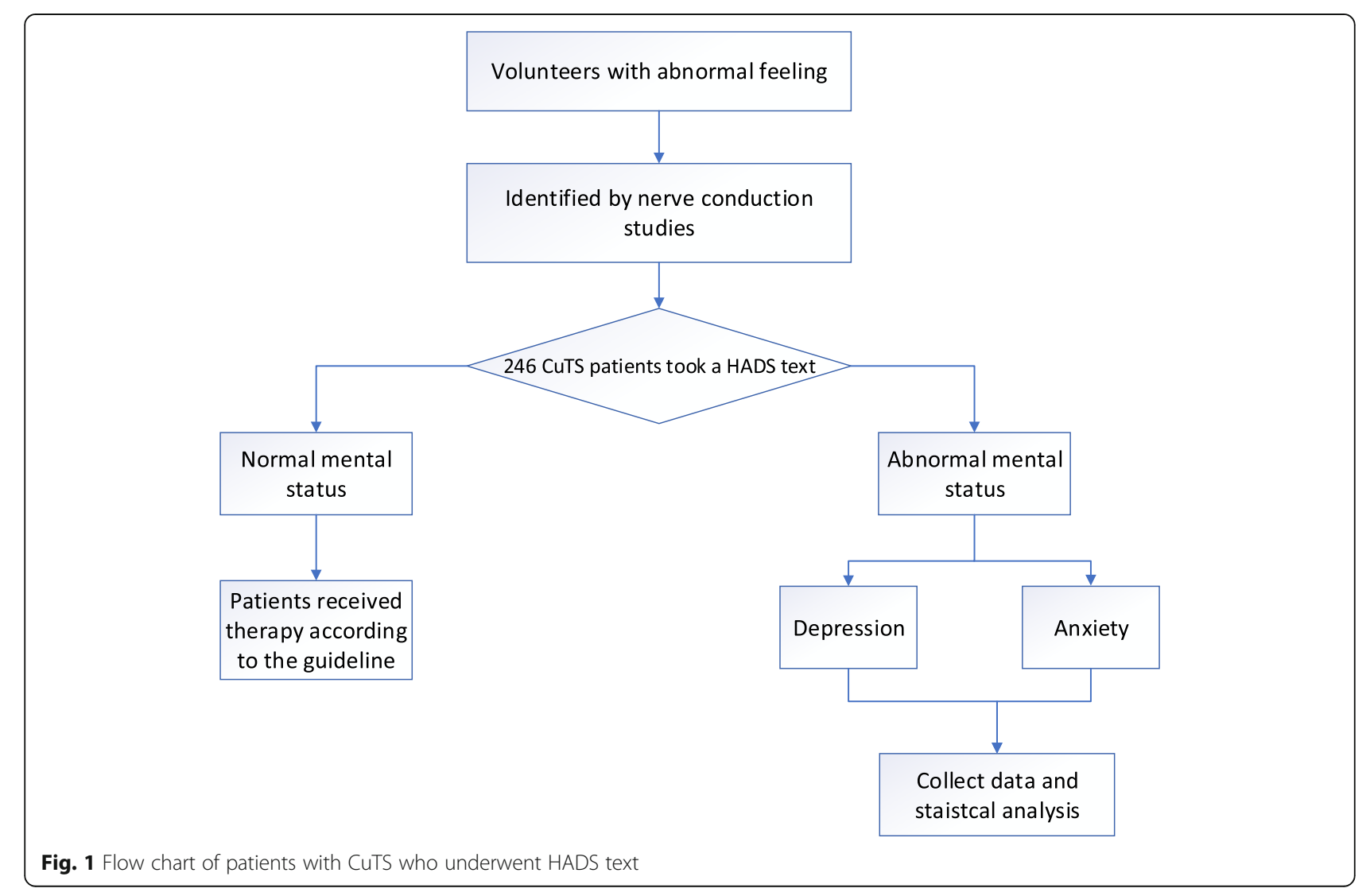


professional group. The criteria of classifying occupation were shown as follows: unskilled occupation: work not requiring education/training - eg: peon, watchman, domestic servant, laborer; semiskilled occupation: need some training to do the routine job efficiently - eg: laboratory attendant, library attendant; skilled occupation: long training in complicated work - eg: carpenter, mechanic, driver, telephone operator; semi-professional - high school teacher, lecturers in college, junior administrators, research assistants; professional- those involved in decision making, laying down policies and executing them - eg: doctors, senior administrative officers, senior lecturers, readers, professors, principals of colleges, advocates, engineers. The socioeconomic status was recorded based on the total amount of family income (US Dollar/per year) (high > 12, 735; medium $=4126$ to 12,735 ; low <4126) [14]. The duration of symptoms was determined as long ( $>2$ years) and short ( $\leq 2$ years) terms. Based on the standard of World Health Organization, alcohol use was defined as $\geq 60 \mathrm{~g}$ on one occasion in the past 30 days [15]. Patients were asked to complete the Quick Disabilities of the Arm, Shoulder, and Hand (QuickDASH) questionnaire to assess the hand function $(0=$ no disability, $100=$ total disability $)[16,17]$. It is a more quickly administered version of $\mathrm{DASH}$, developed using Rasch analysis [17]. It has adequate convergent and discriminant validity, excellent internal consistency reliability with a Cronbach $\alpha$ of 0.89 in the primary care setting [18]. Based on the modified McGowan grade [10], the patients were classified into four groups (grade I: subjective symptoms, no abnormal objective findings; grade IIa: good intrinsic strength (4/5), no detectable muscle atrophy; grade IIb: fair intrinsic strength (3/5), detectable muscle atrophy;
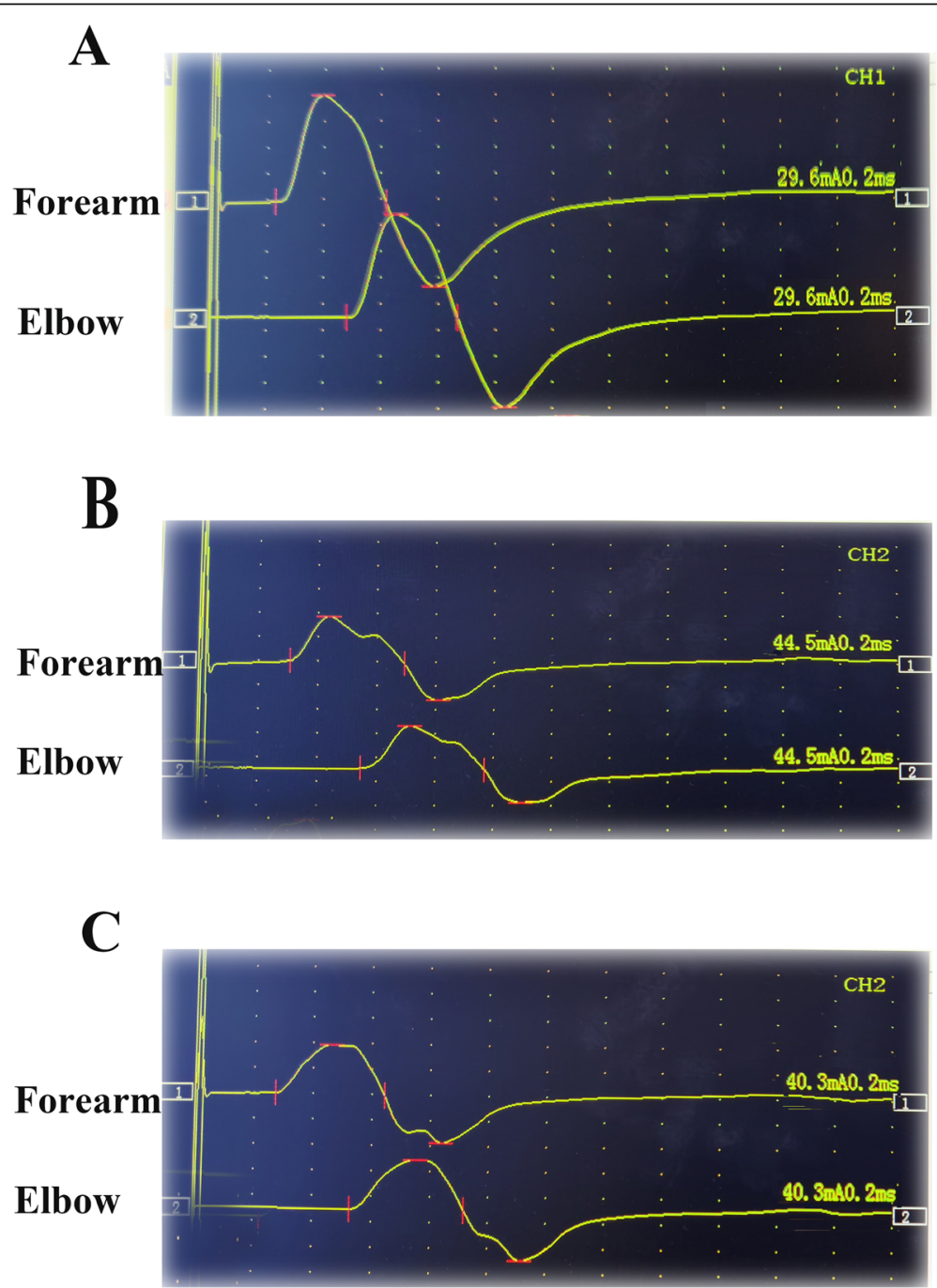

Fig. 2 The picture of sample electrodiagnostic evidence of CUTS. a Patient with CuTS but without depression and anxiety symptoms. b Patient with both CUTS and depression symptoms. c Patient with both CuTS and anxiety symptoms 
grade III: profound sensory and motor disturbances with marked intrinsic atrophy). We used the Chinese version Hospital Anxiety and Depression Scale (HADS) ( $\geq 11$ points, probable disorder; 8 to 10 , possible cases; and $\leq 7$, no case) to determine the anxiety and depression [19]. The HADS (21-item) contains anxiety sub-scale (HADS-A) and depression sub-scale (HADS-D) with each including 7 questions. We classified the patients as being depressed or anxious (present case, $\geq 8$ points) or nondepressed/nonanxious (absent case, $\leq 7$ points). The cut-off scores of depression and anxiety were 8 points. In China, the validated HADS is commonly used for interviewing participants. The Chinese version of HADS demonstrated the similar satisfactory linguistic equivalence, conceptual equivalence, and scale equivalence (concordance rates at the cutoff of 8 for anxiety and depression sub-scales were 89 and $87 \%$, respectively; and at the cutoffs of 11 were 87 and 91\%, respectively) compared with the English version [20].

\section{Data collection}

Data was collected by a clinical psychologist (XS) who filled out the pre-coded structured questionnaire that comprised demographic and clinical data collected from the patients.

\section{Statistical analyses}

The main outcomes were the proportions of anxiety and depression, as well as the associated factors. Associations between potential prognostic determinants and outcomes were examined using univariate logistic regression analysis. Predictors univariately associated with outcome $(p<0.05)$ were included in a multiple-predictor logistic regression model. Then multiple logistic regression (Backword-Wald) was carried out to identify the variables that were independently associated with anxiety and depression. Logistic regression was expressed as odds ratios with a 95\% confidence interval (CI). Statistical analyses were performed using the Statistical Package for the Social Sciences (SPSS, version 25, Chicago, IL) for windows.

\section{Results}

A total of 295 patients with typical electromyographic images of MNCV across the elbow $(<50 \mathrm{~m} / \mathrm{s})$ were recruited (Fig. 2). We excluded 49 patients because they refused to participate. Finally, 246 patients were included in this study with response rate of $83 \%$. The mean age was $63.0 \pm 3.8$ years. There were $180(73 \%)$ female patients; $166(68 \%)$ patients had primary or middle education; 101 (41\%) patients were in the medium socioeconomic level; 175 (71\%) patients were married; 18 (7\%) patients reported tobacco use; and 23 (9\%) patients reported alcohol use. The most frequent medical comorbidities were diabetes mellitus $(18 \%, n=44)$ and hypertension $(11 \%, n=26)$. The average duration of symptoms was $23.9 \pm 4.1$ months. Based on modified McGowan grade, all patients were classified into
Table 1 Demographic and clinical characteristics of study sample $(N=246)$

\begin{tabular}{|c|c|}
\hline Characteristics & \\
\hline Age (year) & $63.1+3.8$ \\
\hline \multicolumn{2}{|l|}{ Gender, n (\%) } \\
\hline Male & $66(26.8)$ \\
\hline Female & $180(73.2)$ \\
\hline \multicolumn{2}{|l|}{ Marital status, n (\%) } \\
\hline Married & $175(71.1)$ \\
\hline Single & $1(0.4)$ \\
\hline Divorced & $57(23.1)$ \\
\hline Widowed & $13(5.2)$ \\
\hline \multicolumn{2}{|l|}{ Education, n (\%) } \\
\hline University & $55(22.3)$ \\
\hline Primary and middle & $166(67.5)$ \\
\hline Illiterate & $25(10.1)$ \\
\hline \multicolumn{2}{|l|}{ Job status, n (\%) } \\
\hline Unemployed (\%) & $31(12.6)$ \\
\hline Employed & $215(87.4)$ \\
\hline \multicolumn{2}{|l|}{ Socioeconomic status, n (\%) } \\
\hline High & $24(9.7)$ \\
\hline Medium & $101(41.1)$ \\
\hline Low & $121(49.2)$ \\
\hline Tobacco use, n (\%) & $18(7.3)$ \\
\hline Exceeding permitted alcohol limit, n (\%) & $23(9.3)$ \\
\hline Hypertension, n (\%) & $44(17.9)$ \\
\hline Diabetes mellitus, n (\%) & $26(10.6)$ \\
\hline History of cancer, $\mathbf{n}(\%)$ & $1(0.4)$ \\
\hline Duration of symptoms (months) & $23.9 \pm 4.1$ \\
\hline QuickDASH score & $41 \pm 4$ \\
\hline \multicolumn{2}{|l|}{ Modified McGowan grade, n (\%) } \\
\hline Grade I CTS & $88(35.8)$ \\
\hline Grade Ila CTS & $45(18.2)$ \\
\hline Grade Ilb CTS & $59(23.9)$ \\
\hline Grade III CTS & $54(21.9)$ \\
\hline
\end{tabular}

grade I $(n=88)$, grade IIa $(n=45)$, grade IIb $(n=59)$, and grade III $(n=54)$ CuTS. The mean QuickDASH score was $41 \pm 4$. The demographic and clinical characteristics are summarized in Table 1.

The results of the HADS demonstrated that 44 (17.8\%) patients presented with possible/probable depression, and $35(14.2 \%)$ patients presented with possible/probable anxiety. Five patients had both possible/ probable anxiety and depression.

The factors associated with depression were education $(p=0.001)$, job status $(p=0.003)$, Modified McGowan grade $(p=0.003)$ and diabetes mellitus $(p<0.001)$. The factors associated with anxiety were diabetes mellitus $(p=0.024)$, modified McGowan grade $(p=0.011)$, and hypertension $(p=0.024)$. The results of univariate logistic 
Table 2 Demographic and clinical factors association with anxiety and depression among CuTS patients

\begin{tabular}{|c|c|c|c|c|c|c|c|}
\hline & \multirow{2}{*}{$\begin{array}{l}\mathrm{N} \\
\text { Prese }\end{array}$} & \multicolumn{2}{|c|}{ Depression } & \multirow[b]{2}{*}{$P$ value } & \multicolumn{2}{|l|}{ Anxiety } & \multirow[b]{2}{*}{$P$ value } \\
\hline & & $t(n=44)$ & Absent $(n=202)$ & & Present $(n=35)$ & Absent $(n=211)$ & \\
\hline \multicolumn{8}{|l|}{ Demographic characteristics } \\
\hline \multicolumn{8}{|l|}{ Age, n (\%) } \\
\hline$>50$ years old & 181 & $31(70.5)$ & $150(74.3)$ & & $28(80)$ & $153(72.5)$ & \\
\hline$\leqq 50$ years old & 65 & $13(29.5)$ & $52(25.7)$ & 0.604 & $7(20)$ & $58(27.5)$ & 0.352 \\
\hline \multicolumn{8}{|l|}{ Gender, n (\%) } \\
\hline Male & 66 & $16(36.4)$ & $50(24.8)$ & & $5(14.3)$ & $61(28.9)$ & \\
\hline Female & 180 & $28(63.6)$ & $152(75.2)$ & 0.115 & $30(85.7)$ & $150(71.1)$ & 0.071 \\
\hline \multicolumn{8}{|l|}{ Marital status, n (\%) } \\
\hline Married & 175 & $30(68.2)$ & $145(71.8)$ & & $23(65.7)$ & $152(72.0)$ & \\
\hline Single & 1 & 0 & $1(0.5)$ & & 0 & $1(0.4)$ & \\
\hline Divorced & 57 & $10(22.7)$ & $47(23.3)$ & & $9(25.7)$ & $47(22.2)$ & \\
\hline Widowed & 13 & $4(9.1)$ & $9(4.5)$ & 0.624 & $3(8.5)$ & $10(4.7)$ & 0.733 \\
\hline \multicolumn{8}{|l|}{ Education, n (\%) } \\
\hline University & 55 & $19(43.2)$ & $36(17.8)$ & & $6(17.1)$ & $49(23.2)$ & \\
\hline Primary and middle & 166 & $21(47.7)$ & $146(71.8)$ & & $25(71.4)$ & $141(66.8)$ & \\
\hline Illiterate & 25 & $4(9.1)$ & $21(10.4)$ & $0.001^{*}$ & $4(11.4)$ & $21(9.9)$ & 0.721 \\
\hline \multicolumn{8}{|l|}{ Job status, n (\%) } \\
\hline Unemployed (\%) & 31 & $12(27.3)$ & $19(9.4)$ & & $5(14.3)$ & $26(12.3)$ & \\
\hline Unskilled (\%) & 35 & $9(20.5)$ & $26(12.9)$ & & $4(11.4)$ & $31(14.7)$ & \\
\hline Semiskilled/Skilled (\%) & 147 & $19(43.2)$ & $128(63.4)$ & & $19(54.3)$ & $128(60.7)$ & \\
\hline Semi-professional/Professional (\%) & 33 & $4(9.1)$ & $29(14.4)$ & $0.003^{*}$ & $7(20)$ & $26(12.3)$ & 0.606 \\
\hline \multicolumn{8}{|l|}{ Socioeconomic status, n (\%) } \\
\hline High & 24 & $8(18.2)$ & $16(7.9)$ & & $3(8.6)$ & $21(10)$ & \\
\hline Medium & 101 & $15(34.1)$ & $86(42.6)$ & & $10(28.6)$ & $91(43.1)$ & \\
\hline Low & 121 & $21(47.7)$ & $100(49.5)$ & 0.103 & $22(62.9)$ & 99 (46.9) & 0.206 \\
\hline \multicolumn{8}{|l|}{ Clinical characteristics } \\
\hline Diabetes mellitus, n (\%) & 44 & $18(40.9)$ & $26(12.9)$ & $<0.001^{*}$ & $11(31.4)$ & $33(15.6)$ & $0.024^{*}$ \\
\hline Hypertension, n (\%) & 26 & $7(15.9)$ & $19(9.4)$ & 0.204 & $8(22.9)$ & $18(8.5)$ & $0.011^{*}$ \\
\hline Tobacco use, n (\%) & 18 & $4(9.1)$ & $14(6.9)$ & 0.618 & $4(11.4)$ & $14(6.6)$ & 0.313 \\
\hline Alcohol use, n (\%) & 23 & $6(13.6)$ & $17(8.4)$ & 0.281 & $4(11.4)$ & $19(9.0)$ & 0.648 \\
\hline History of cancer, n (\%) \# & 1 & 0 & $1(0.4)$ & 0.75 & $1(2.8)$ & 0 & 0.85 \\
\hline \multicolumn{8}{|l|}{ Duration of symptoms, n (\%) } \\
\hline$>2$ years & 124 & $25(56.8)$ & $99(49)$ & & $18(60)$ & $106(37.6)$ & \\
\hline$\leqq 2$ years & 122 & $19(43.2)$ & $103(51)$ & 0.348 & $17((40)$ & $105(62.4)$ & 0.896 \\
\hline QuickDASH score & & $41 \pm 4$ & $42 \pm 3$ & 0.061 & $40 \pm 5$ & $41 \pm 3$ & 0.082 \\
\hline \multicolumn{8}{|l|}{ Modified McGowan grade, n (\%) } \\
\hline Grade I CuTS & 88 & $24(54.5)$ & $64(33.7)$ & & $14(40)$ & $74(35.1)$ & \\
\hline Grade Ila CuTS & 45 & 0 & $45(22.3)$ & & 0 & $45(21.3)$ & \\
\hline Grade Ilb CuTS & 59 & $11(25)$ & $48(23.8)$ & & $12(34.3)$ & $47(22.3)$ & \\
\hline Grade III CuTS & 54 & $9(20.5)$ & $45(22.3)$ & $0.003^{*}$ & $9(25.7)$ & $45(21.3)$ & $0.021^{*}$ \\
\hline
\end{tabular}

Chi-squared test was used, $* P<0.05$, \# Fishers exact test was used 
Table $\mathbf{3}$ Logistic regression for variables associated with anxiety and depression

\begin{tabular}{llllll}
\hline & Variable & $\boldsymbol{\beta}$ & Odds ratio & $\mathbf{9 5 \%} \mathrm{Cl}$ & $\mathbf{p}$ value \\
\hline Depression & Job status & -0.46 & 1.584 & $0.802-3.128$ & 0.185 \\
& Education & -0.365 & 0.694 & $0.209-2.306$ & $0.457-1.193$ \\
& Modified McGowan grade & -0.304 & 0.738 & $1.650-18.806$ & 0.215 \\
Anxiety & Diabetes Mellitus & 1.717 & 5.570 & $0.378-0.911$ & $0.006^{*}$ \\
& Modified McGowan grade & -0.533 & 0.587 & $0.499-9.894$ & 0.295 \\
& Diebettes millitus & 0.799 & 2.222 & $0.804-19.568$ \\
\hline
\end{tabular}

Multivariable logistic analysis was used, ${ }^{*} P<0.05$

Illiterate; unemployed and Grade III CTS were taken as a reference value in the calculation of Adjusted Odds Ratio for educational status, Job status and Modified McGowan grade

analysis are summarized in Table 2. Multivariable logistic analysis of univariate factors associated with depression and anxiety is shown in Table 3 . Diabetes mellitus was independently associated with depression $(p=0.006)$. Modified McGowan grade was significantly associated with anxiety $(p=0.017)$.

\section{Discussion}

Many studies have shown that chronic diseases, such as diabetes and asthma, can cause mental health disorders after a prolonged period of time [21]. Beleckas et al. [13] found that the CuTS-related proportions of depressive and anxiety disorders were 16 and $9 \%$, respectively, based on the National Institutes of Health developed the Patient-Reported Outcomes Measurement Information System. Anxiety may last for a long time before the medical intervention. Until now, few studies focus on the prevalence of depression and anxiety in patients with CuTS.

We found $18 \%$ of patients had significant symptoms of possible/probable depression disorder, and $14 \%$ had the symptoms of the possible/probable depressive disorder. Similarly, Belecka et al. [9] also found that patients with upper extremity conditions more frequently reported anxiety and depression than the general population did. Those findings are helpful for early psychiatric evaluation and identifying the presence of depression and anxiety in patients with CuTS.

Logistic regression analysis revealed that diabetes mellitus is independently associated with depression. Diabetes mellitus can cause small fiber neuropathy, which forms a significantly painful condition in a patient's body [22]. Therefore, it is possible that the small fiber neuropathy worsens the symptoms of CuTS, and the cooperative effect increases the risk of depression.

In our study, the modified McGowan grade was independently associated with anxiety. We found a higher proportion of anxiety in patients with grade IIb $(20 \%)$ and III (17\%) CuTS, compared with that in patients with grade I (16\%) and IIa (0) CuTS. Patients with anxiety tend to report worse hand function, and the worse hand function worsen their anxiety symptoms. This finding showed that worse anxiety is associated with higher symptom burden and worse physical functioning. Therefore, early intervention is beneficial to stop the progression of the disease for patients with mental health disorders.

Our study has limitations. The sample size is small, which affects the comprehension of the questionnaires presented to those participants. There is a bias that the HADS is a screening tool for assessing anxiety and depression rather than a clinical diagnostic tool. Our study is based on one time point and is thus cross-sectional. Therefore, we are unable to determine the causal relationships or establish the direction of associations.

\section{Conclusion}

In patients with $\mathrm{CuTS}$, the proportions of depression and anxiety were 18 and 14\%, respectively. Early screening for anxiety and depression is beneficial for patients with CuTS.

\section{Abbreviations \\ CUTS : Cubital tunnel syndrome; QuickDASH: Quick disabilities of the arm, shoulder, and hand; HADS: Hospital anxiety and depression scale; \\ Cl: Confidence interval; SPSS: Statistical package for the social sciences}

\section{Acknowledgements}

We are particularly grateful to all the people who have given us help on our article.

\section{Authors' contributions}

(I) Conception and design: XS, SJ. (II) Administrative support: XS, SJ. (III) Provision of study materials or patients: GL, LW. (IV) Collection and assembly of data: XZ, XM. (V) Data analysis and interpretation: XZ, XM, JL. (VI) Manuscript writing: All authors(VII) Final approval of manuscript: All authors. All authors have read and approved the manuscript", and ensure that this is the case.

\section{Funding}

This work was supported by the Chinese Traditional and Western Medicine Clinical Collaborative Pilot Project for Major Difficult Diseases of the National Administration of Traditional Chinese Medicine "Degenerative Osteoarthritis of the Elbow Joint with Ulnar Nerve Entrapment Syndrome". The funder was not involved in the design of the study and collection, analysis, and interpretation of data and in writing the manuscript of this study. 


\section{Availability of data and materials}

The datasets used and/or analysed during the current study available from the corresponding author on reasonable request.

\section{Ethics approval and consent to participate}

The authors are accountable for all aspects of the work in ensuring that questions related to the accuracy or integrity of any part of the work are appropriately investigated and resolved.This study complied with the Declaration of Helsinki and was approved by Ethics Committees of the Third Hospital of Hebei medical university. Except for illiterate patients,all patients provided written informed consent. Illiterate patients indicate their consent by"making their mark** on the consent form, which were consistent with the local law.

\section{Consent for publication}

Not Applicable.

\section{Competing interests}

The authors declare that they have no conflict of interest.

\section{Author details}

'Department of Hand Surgery, The Third hospital of Hebei Medical University, Shijiazhuang 050000, Hebei, China. ${ }^{2}$ Graduate School, Hebei Medical University, Shijiazhuang 050000, Hebei, China. ${ }^{3}$ Department of Neurology, Hebei General Hospital, Shijiazhuang 050000, Hebei, China. ${ }^{4}$ Department of Bone Surgery, Hebei Pingshan General Hospital, Shijiazhuang 050000, Hebei, China. ${ }^{5}$ Department of Hand Surgery, Xuzhou Renci Hospital, Xuzhou 221000, Jiangsu, China.

Received: 21 April 2020 Accepted: 27 October 2020

Published online: 17 November 2020

\section{References}

1. Zhu L, Zhi RJ, Ting TW, Xin S, Wei L. Preliminary study on the lesion location and prognosis of cubital tunnel syndrome by motor nerve conduction studies. Chin Med J. 2015;128(9):1165-70. https://doi.org/10.4103/0366-6999. 156100.

2. Andrews K, Rowland A, Pranjal A, Ebraheim N. Cubital tunnel syndrome: anatomy, clinical presentation, and management. J Orthop. 2018;15(3):8326. https://doi.org/10.1016/j.jor.2018.08.010.

3. Osei DA, Groves AP, Bommarito K, Ray WZ. Cubital tunnel syndrome: incidence and demographics in a national administrative database. Neurosurgery. 2017;80(3):417-20. https://doi.org/10.1093/neuros/nyw061.

4. Blok RD, Becker SJE, Ring DC. Carpal tunnel syndrome: clinical features, diagnosis, and management. Lancet Neurol. 2016;15(12):1273-84. https:// doi.org/10.1016/S1474-4422(16)30231-9.

5. Kyle A, Andrea R, Ankur P, Nabil E. Cubital tunnel syndrome: Anatomy, clinical presentation, and management. J Orthop. 2018;15(3):832-6. https:// doi.org/10.1016/j.jor.2018.08.010.

6. Rachel HS, Janet SH, Lyn YA. Gender differences in depression in representative national samples: meta-analyses of diagnoses and symptoms. Psychol Bull. 2017;143(8):783-822. https://doi.org/10.1037/bul0000102.

7. Scott BP. The "Clinician's illusion" and the epidemiology, diagnosis and treatment of depressive disorders. BMC Psychiatry. 2018;18(1):395. https:// doi.org/10.1186/s12888-018-1969-3.

8. Jamshidi AR, Banihashemi AT, Paragomi P, Hasanzadeh M, Barghamdi M Ghoroghi S. Anxiety and depression in rheumatoid arthritis: an epidemiologic survey and investigation of clinical correlates in Iranian population. Rheumatol Int. 2016;36(8):1119-25. https://doi.org/10.1007/ s00296-016-3493-4.

9. Beleckas CM, Wright M, Prather H, Chamberlain A, Guattery J, Calfee RP. Relative prevalence of anxiety and depression in patients with upper extremity conditions. J Hand Surg Am. 2018;43(6):571.e571-8. https://doi. org/10.1016/j.jhsa.2017.12.006.

10. Krejčí T, Večeřa Z, Krejčí O, Šalounová D, Houdek M, Lipina R. Comparing endoscopic and open decompression of the ulnar nerve in cubital tunnel syndrome: a prospective randomized study. Acta Neurochir. 2018;160(10): 2011-7. https://doi.org/10.1007/s00701-018-3647-0.

11. Van SM, Moons KG, de GJA, Collins GS, Altman DG, Eijkemans MJ, Reitsma JB. Sample size for binary logistic prediction models: beyond events per variable criteria. Stat Methods Med Res. 2019;28(8):2455-74. https://doi.org/ 10.1177/0962280218784726.

12. Kiril EBV, Michel W, Viviane vK. Ultrasonography and electrodiagnostic studies in ulnar neuropathy: an examination of the sensitivity and specificity and the correlations between both diagnostic tools. Clin Neurophysiol. 2015;32(3):240-3. https://doi.org/10.1097/WNP.0000000000000148.

13. Nakić Radoš S, Tadinac M, Herman R. Anxiety during pregnancy and postpartum: course, predictors and comorbidity with postpartum depression. Acta Clin Croat. 2018;57(1):39-51. https://doi.org/10.20471/acc. 2017.56.04.05.

14. Bank World. World development report, 2015. Accessed from https:// elibrary.worldbank.org/doi/abs/10.1596/978-1-4648-0342-0.

15. Peacock A, Leung J, Larney S, Colledge S, Hickman M, Rehm J, Giovino GA, West R, Hall W, Griffiths P, et al. Global statistics on alcohol, tobacco and illicit drug use: 2017 status report. Addiction. 2018;113(10):1905-26. https:// doi.org/10.1111/add.14234

16. Joy CM, Leenesh K, Trevor BB, George SA. Validity of the QuickDASH in patients with shoulder-related disorders undergoing surgery. J Orthop Sports Phys Ther. 2015;45(1):25-36. https://doi.org/10.2519/jospt.2015.5033.

17. Gummesson C, Ward MM, Atroshi I. The shortened disabilities of the arm, shoulder and hand questionnaire (QuickDASH): validity and reliability based on responses within the full-length DASH. BMC Musculoskelet Disord. 2006; 7(1):44-0. https://doi.org/10.1186/1471-2474-7-44.

18. Hammond A, Prior $Y$, Tyson S. Linguistic validation, validity and reliability of the British English versions of the disabilities of the arm, shoulder and hand (DASH) questionnaire and QuickDASH in people with rheumatoid arthritis. BMC Musculoskelet Disord. 2018;19(1):118. https://doi.org/10.1186/s12891018-2032-8.

19. Zigmond AS, Snaith RP. The hospital anxiety and depression scale. Acta Psychiatr Scand. 1983;67(6):361-70. https://doi.org/10.1111/j.1600-0447.1983. tb09716.x.

20. Leung $\mathrm{CM}$, Ho S, Kan CS, Hung $\mathrm{CH}$, Chen CN. Evaluation of the Chinese version of the hospital anxiety and depression scale. A cross-cultural perspective. Int J Psychosom. 1993;40(1-4):29-34.

21. Tasneem K, Christopher MC, Anthony JP, Jesse CS. Depression and anxiety screens as simultaneous predictors of 10-year incidence of diabetes mellitus in older adults in primary care. J Am Geriatr Soc. 2017;65(2):294-300. https:// doi.org/10.1111/jgs.14454.

22. Weidemann M, Reichmann H, Ziemssen T, Buchmann S, Illigens B, Siepmann T. Autonome small-fiber-neuropathien. Neurologie. 2019. https:// doi.org/10.1055/a-0794-9314

\section{Publisher's Note}

Springer Nature remains neutral with regard to jurisdictional claims in published maps and institutional affiliations.

Ready to submit your research? Choose BMC and benefit from:

- fast, convenient online submission

- thorough peer review by experienced researchers in your field

- rapid publication on acceptance

- support for research data, including large and complex data types

- gold Open Access which fosters wider collaboration and increased citations

- maximum visibility for your research: over $100 \mathrm{M}$ website views per year

At $\mathrm{BMC}$, research is always in progress.

Learn more biomedcentral.com/submissions 